\title{
Liver Volumetry Plug and Play: Do It Yourself with ImageJ
}

\author{
Simon A. W. G. Dello · Ronald M. van Dam · Jules J. G. Slangen • \\ Marcel C. G. van de Poll · Marc H. A. Bemelmans · Jan Willem W. M. Greve • \\ Regina G. H. Beets-Tan · Stephen J. Wigmore $\cdot$ Cornelis H. C. Dejong
}

Published online: 29 August 2007

(C) Société Internationale de Chirurgie 2007

\begin{abstract}
Background A small remnant liver volume is an important risk factor for posthepatectomy liver failure and can be predicted accurately by computed tomography (CT) volumetry using radiologic image analysis software. Unfortunately, this software is expensive and usually requires support by a radiologist. ImageJ is a freely downloadable image analysis software package developed by the National Institute of Health (NIH) and brings liver volumetry to the surgeon's desktop. We aimed to assess the accuracy of ImageJ for hepatic CT volumetry.

Methods ImageJ was downloaded from http://www.rsb. info.nih.gov/ij/. Preoperative CT scans of 15 patients who
\end{abstract}

Authorship statement; Simon A. W. G. Dello and Ronald M. van Dam contributed equally to the realization of the study and the manuscript and share first authorship.

S. A. W. G. Dello · R. M. van Dam · J. J. G. Slangen ·

M. C. G. van de Poll - M. H. A. Bemelmans

J. W. W. M. Greve · C. H. C. Dejong

Department of Surgery, University Hospital, Maastricht,

The Netherlands

R. G. H. Beets-Tan

Department of Radiology, University Hospital, Maastricht,

The Netherlands

S. J. Wigmore

Liver Unit, Queen Elizabeth Hospital, Birmingham, UK

M. C. G. van de Poll - J. W. W. M. Greve · C. H. C. Dejong Nutrition and Toxicology Research Institute (NUTRIM),

Maastricht University, Maastricht, The Netherlands

C. H. C. Dejong $(\varangle)$

Department of Surgery, Maastricht University, PO Box 616, 6200 MD, Maastricht, The Netherlands

e-mail: C.Dejong@surgery.azm.nl underwent liver resection for colorectal cancer liver metastases were retrospectively analyzed. Scans were opened in ImageJ; and the liver, all metastases, and the intended parenchymal transection line were manually outlined on each slice. The area of each selected region, metastasis, resection specimen, and remnant liver was multiplied by the slice thickness to calculate volume. Volumes of virtual liver resection specimens measured with ImageJ were compared with specimen weights and calculated volumes obtained during pathology examination after resection.

Results There was an excellent correlation between the volumes calculated with ImageJ and the actual measured weights of the resection specimens $\left(r^{2}=0.98, p<0.0001\right)$. The weight/volume ratio amounted to $0.88 \pm 0.04$ (standard error) and was in agreement with our earlier findings using CT-linked radiologic software.

Conclusion ImageJ can be used for accurate hepatic CT volumetry on a personal computer. This application brings CT volumetry to the surgeon's desktop at no expense and is particularly useful in cases of tertiary referred patients, who already have a proper CT scan on CD-ROM from the referring institution. Most likely the discrepancy between volume and weight results from exsanguination of the liver after resection.

The incidence of colorectal cancer (CRC) is steadily increasing in the Western world [1,2]. Approximately 50\% of all patients diagnosed with CRC develop liver metastases at some stage of their disease. For these patients, liver resection is the only potentially curative treatment option. The number of candidates for liver resection is limited, however. One of the reasons is that liver dysfunction may occur when the extent of tumor involvement requires a 
major resection, leaving a small postoperative remnant liver volume [3-5].

As we [3, 6] and others [7] have shown before, pre- and postoperative liver volumes can be accurately calculated from computed tomography (CT) or magnetic resonance imaging (MRI) scans. Liver volumetry is useful for patient selection and helps reduce the incidence of complications due to insufficient residual liver volume [3, 5, 8, 9]. Unfortunately, radiologic image analysis software is typically linked to radiologic hardware, making it less accessible for nonradiologists. In addition, the intended operation should be known to the investigator to predict the remnant liver volume accurately. This requires the expertise of a liver surgeon. Therefore, CT volumetry has hitherto been a multidisciplinary modality requiring the efforts of dedicated surgeons and radiologists. Advances in digitalization, the availability of broadband networks, and the introduction of CT scans on CD-ROM have enabled volumetry on a personal computer remote from radiological hardware (CT scanners or MRI). Advantages of standalone software are its applicability for tertiary referred patients, who already have a proper CT scan on CD-ROM from the referring institution and the possibility of performing liver volumetry independent of the input of a radiologist. However, commercially available stand-alone CT volumetry software is often expensive. Recently, an alternative approach using Adobe Photoshop was proposed to circumvent this problem, but the method is laborious [7]. Image $\mathrm{J}$ is a freely downloadable image analysis software package developed at the National Institute of Health (NIH) to assist in clinical and scientific image analyses. The applicability of ImageJ for liver volumetry has not been addressed before, but it potentially brings liver volumetry to the surgeon's desktop [10]. The objective of the present study was to establish the accuracy of ImageJ for CT volumetric analysis of the liver on a personal computer in patients undergoing major liver resection for CRC metastases.

\section{Material and methods}

Patients, CT scans, liver weight

In 2000, a prospective database was created in which data on all patients undergoing liver resection in our institution were collected. From this database, all patients who underwent major liver resection ( $\geq 3$ segments) for colorectal metastases $(n=70)$ between 2000 and 2005 were selected. Subsequently, pathology files of these patients were checked by one of the authors (R.M.V.D.) to detect those cases in which liver specimens had been weighed by the pathologist. This yielded 15 patients (Table 1) who
Table 1 Patient characteristics

\begin{tabular}{ll}
\hline Parameter & Results \\
\hline Age (years) & $60(34-77)$ \\
Sex (M/F) & $7 / 8$ \\
Height (cm) & $173(160-186)$ \\
Body weight (kg) & $75(55-110)$ \\
Right hepatectomy (no.) & 12 \\
Left hepatectomy (no.) & 3 \\
Wedge resection (VII) (no.) & 1 \\
Segmentectomy (III) (no.) & 1 \\
AST (IU/L) & $71(14-443)$ \\
ALT (IU/L) & $69(14-392)$ \\
LDH (IU/L) & $554(250-1617)$ \\
$\gamma$-Glutamyltranspeptidase (IU/L) & $43(6-112)$ \\
Alkaline phosphatase (IU/L) & $109(67-215)$ \\
Bilirubin ( $\mu$ M) & $18.4(9.2-47)$ \\
\hline
\end{tabular}

Results are the median and range or the number

underwent major liver resection with a known specimen weight. Two of these patients underwent an extended procedure: one left hepatectomy + wedge resection and one right hepatectomy + segmentectomy.

Patients underwent CT scanning in their routine preoperative assessment either in our hospital or in one of the surrounding university-affiliated district general teaching hospitals. For volumetric analysis, four-phase CT scans were used that were provided on CD-ROM on four different viewers: eFilm Lite (eFilm Medical. Toronto, Canada), SIENET MagicView 300 VA42D (Siemens, Erlangen, Germany), DICOM CDViewer 3.412 (Quazar Software GmbH, Hamburg, Germany), or DICOM LiteBox, version 2.02C (Rennes, France). The volumetric analyses were performed by two other investigators (S.A.W.G.D., J.J.G.S.) who were blinded to the weight of the resected specimens.

Immediately following resection, the resection specimens were transferred to the pathology department by one of the surgeons to scrutinize resection margins. The specimens were weighed immediately upon arrival at that department.

Calculation of liver volume with ImageJ

1. Downloading ImageJ: ImageJ (version 1.33) was downloaded from http://www.rsb.info.nih.gov/ij/ download.html (accession date: 01/07/2005).

2. Making a stack: The portovenous phase of four-phase contrast-enhanced CT scans was used to facilitate optimal identification of liver segments and the anatomic resection plane in the individual patients. Relevant CT slices were evaluted in the original CT viewer. Every CT slice has a unique code or number 
that can be found in the information menu of the CT viewer, matching a JPEG file. The JPEG files were retrieved with Windows Explorer and opened in ImageJ by dragging them to the ImageJ main window. Individual slices were transformed to a "stack" using the function "Convert Images to Stack" in submenu "Stacks" in the pull-down menu "Image" (Fig. 1).

3. Adjusting scale: After opening DICOM images in ImageJ, the scaling of the images is corrected automatically, and volumetric analysis can be continued. However, in non-DICOM viewers, the scale of the imported stack was adjusted by measuring the distance between two randomly chosen but clearly recognizable points on a slice in the original CT viewer using its measurement tool. Subsequently, the line between these points was traced on the corresponding slice and its distance set in ImageJ using the "Set scale" function in the "Analyze" submenu.

4. Creating a region of interest: On the CT slices there were three regions of interest (ROI) relevant for the present study: total liver area, metastasis area, and resection specimen area. Before outlining the ROI on each slice, the ROI manager in the pull-down menu "Analyze", submenu "Tools" was opened. The total liver, metastases, and resection specimen were manually outlined with the "Polygon selection tool." This tool can create an irregularly shaped selection defined by a series of line segments. To create an ROI on the CT slice, the mouse has to be clicked repeatedly to create line segments. When finished, one has to click in the small box at the starting point, and ImageJ automatically draws the last segment. The respective ROI of each slice was added to the ROI manager with the function "Add" in the ROI manager menu. The resection specimen was outlined according to transection planes as described in the operation notes. The gallbladder and the inferior caval vein were excluded from the ROI; intrahepatic biliary and vascular structures were included. In all patients with a hemihepatectomy, the transection line of the real liver resection followed Cantlies line from the top of the gallbladder, paralleling the middle hepatic vein straight to the suprahepatic inferior cava vein. In all cases the middle hepatic vein stayed in situ with the liver remnant (Fig. 2).

5. Calculating volume: To calculate the areas, all the ROIs must be selected in the ROI manager. The area of each ROI was calculated with the function "Measure" in the ROI manager menu. The calculated areas were then selected and copied (right click) to Microsoft Excel, where the areas were multiplied with slice thickness (ranging from 3 to $7 \mathrm{~mm}$, varying per viewer and/or patient). The last step was to add up these values per slice and calculate the volume of each threedimensional structure. The data are then presented in cubic millimeters. Subsequently, these values per slice were added up to calculate the volume of each threedimensional structure.

Time required to perform CT volumetry and effect of slice thicknesses

To assess the time required to perform liver volumetry with ImageJ, the time needed to outline the three ROIs (total liver, metastases, resection specimen) per slice was recorded in one liver (a liver with one large tumor that required right hemihepatectomy). The time per slice was recorded, and the average time per slice for the entire liver was calculated. In addition, we calculated liver volumes using various slice thicknesses to evaluate the maximum slice thickness at which volumetry was still accurate.

\section{Statistics}

Results are presented as the median and range, as indicated in the Results section. The correlation between the actual weights of the resection specimens (determined during the pathology examination) and corresponding volumes calculated with ImageJ was evaluated with Pearson's test. A value of $p<0.05$ was considered to indicate statistical significance. Statistics were performed using Prism 4.0 for Windows (GraphPad Software, San Diego, CA, USA).

\section{Results}

Patients

Patient characteristics are shown in Table 1. The median age of the patients $(n=15)$ was 60 years (range 34-77 years). None of the patients had any preoperative evidence of underlying liver disease, and routine liver tests were uniformly normal in all but one patient, who had slightly elevated plasma levels of liver enzymes. In total, 14 patients underwent a major anatomic resection. Right hepatectomy was performed in 11 patients, extended with a segmentectomy (segment III) in one. Left hepatectomy was performed in two patients, extended with a metastasectomy in segment VII in one. One patient underwent posterior sectionectomy extended with a segmentectomy of segment 
Fig. 1 ImageJ showing the "making a stack" pull-down menus

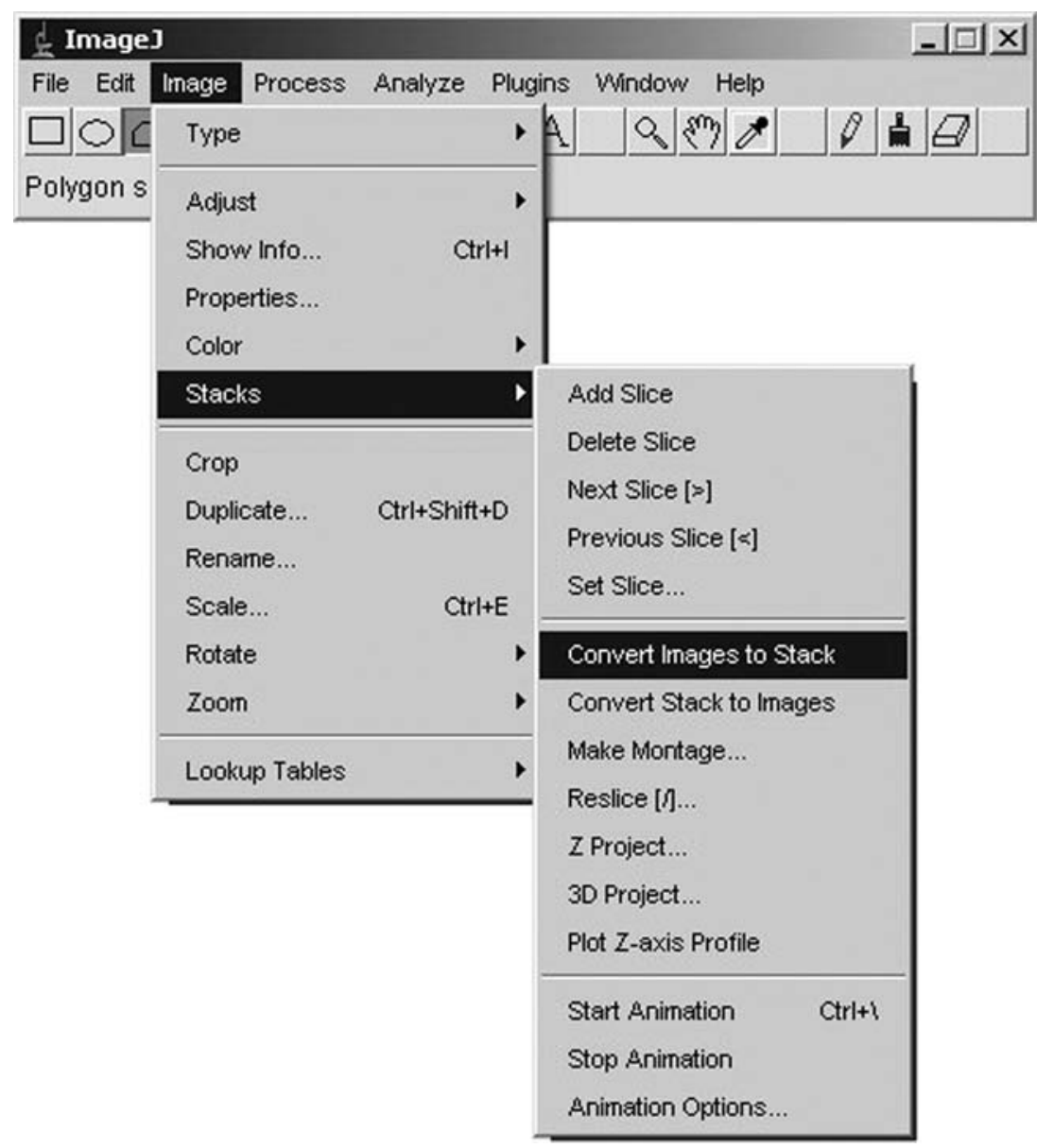

III, and one patient underwent left lateral sectionectomy. There were no postoperative deaths.

Relation between resected liver weight and CTmeasured liver volume

The median resected liver volume calculated with ImageJ was $887 \mathrm{ml}$ (range 20-2173 ml). The median weight of the resection specimens measured during pathology examination was $732 \mathrm{~g}$ (range 23-1957 g). There was a highly significant correlation between resected liver volumes calculated with ImageJ and the actual measured weights of the resection specimens (Fig. 3) $\left(r^{2}=0.98\right.$, $p<0.0001)$. The mean ratio between measured weight and calculated volume of the resection specimens was $0.88 \pm 0.04(\mathrm{SE}) \mathrm{g} / \mathrm{ml}$.

\section{CT-measured total liver volume/weight}

The calculated median total liver volume in our study population was $1610 \mathrm{ml}$ (range 1185-2990 ml), and the median total tumor volume was $63 \mathrm{ml}$ (range 4-1102 ml).
The median functional liver volume (total liver volume tumor volume $=$ normal liver tissue volume) was $1606 \mathrm{ml}$ (range 944-2851 ml).

Time required to perform CT volumetry and effect of slice thickness

To assess the time required to perform volumetry with ImageJ, the time needed to outline the three relevant ROIs (total liver volume, metastases volume, and volume of the resection specimen) was recorded for one liver with a total volume of $1680.1 \mathrm{ml}$ and a metastases volume of $32.8 \mathrm{ml}$ when right hemihepatectomy was performed. There were, in total, 34 slices; and analysis took on average of $50 \pm 3$ seconds per slice. Considering the average time needed per slice, it can be calculated that at a slice thickness of $5 \mathrm{~mm}$ a full volumetric analysis of a single liver requires on average of 25 to 28 minutes. Increasing the slice thickness results in a decline of accuracy of the volumetric assessment (Fig. 4). A maximum CT slice thickness of $1.0 \mathrm{~cm}$ reduces the number of slices needed to define an ROI and adds only a little error in the weight/volume ratio, thus providing an optimal balance between accuracy and time efficiency. 
Fig. 2 Computed tomography (CT) scan opened in Image J showing the region of interest (ROI) pull-down menus and a large tumor in the right hemiliver requiring right hepatectomy. The resection specimen is outlined on every slice as an ROI and added to the ROI manager (bottom right)

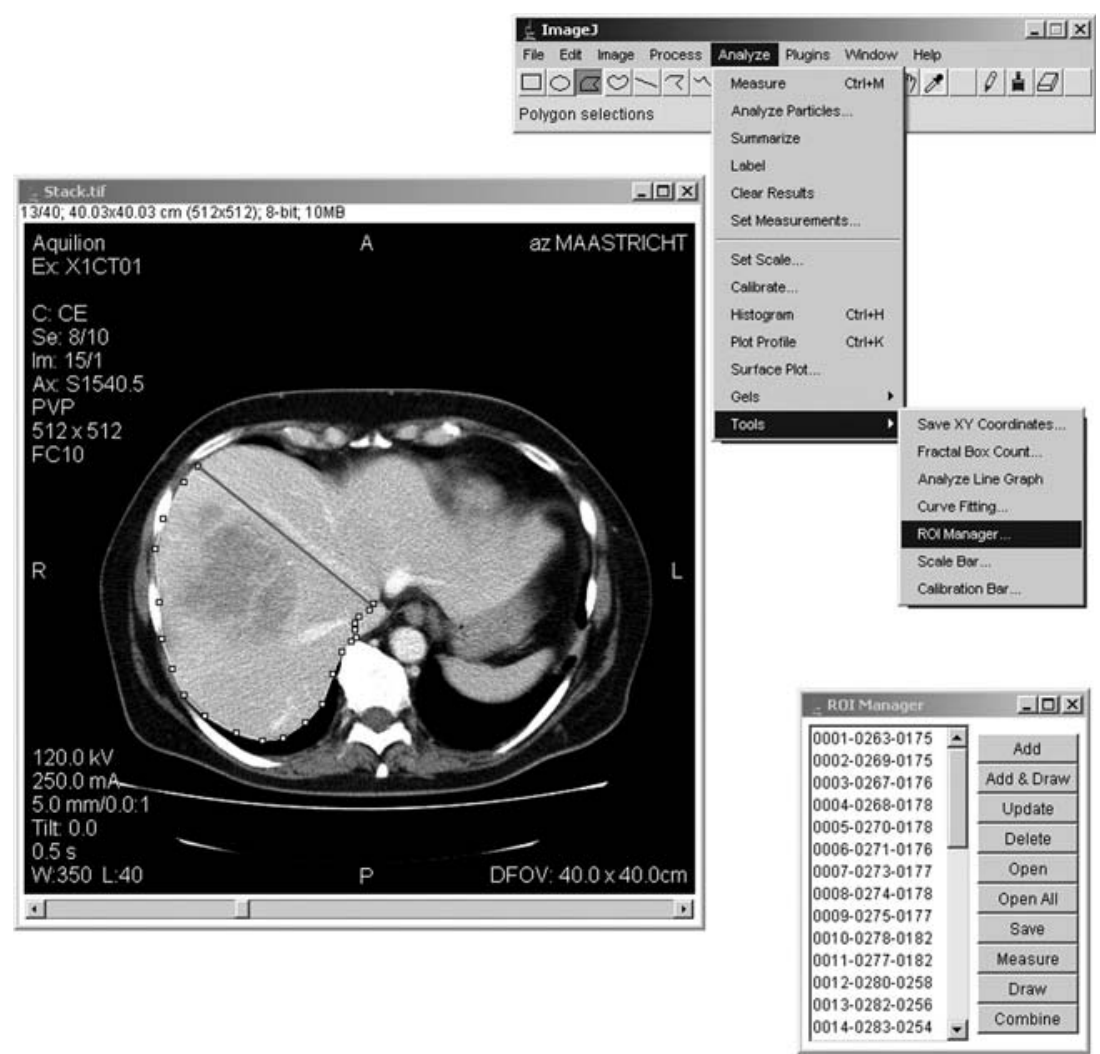

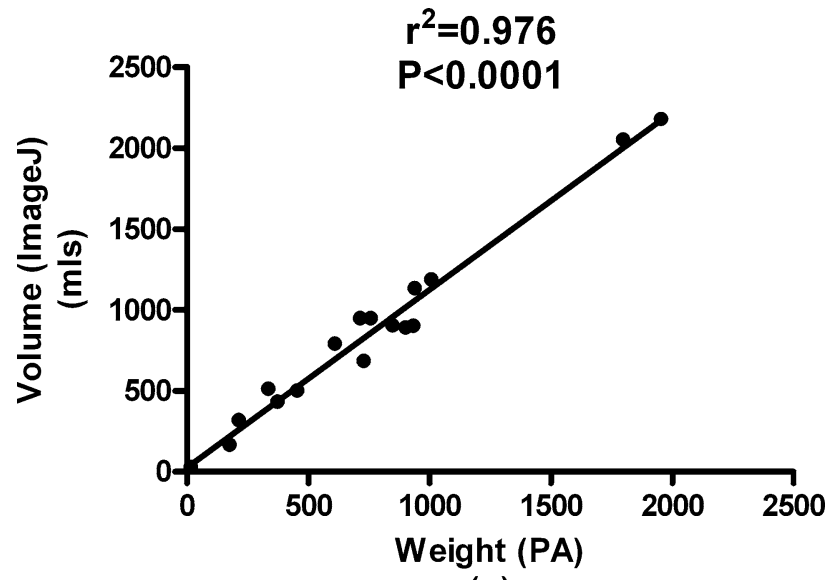

(g)

Fig. 3 Correlation between volume of resection calculated with Image J and actual measured weights of the resection specimens $(n=15$, Pearson's test $)$

\section{Discussion}

In this study, we aimed to assess the accuracy of the freely downloadable image analysis software package ImageJ for hepatic CT volumetry in patients undergoing major hepactectomy for colorectal cancer metastases. With the use of ImageJ, an accurate CT volumetric analysis can be performed on a personal computer, bringing liver volumetry within the surgeon's reach, independent of the support of a radiologist. The results are in agreement with our previous experience using professional radiological software [3, 6], supporting the accuracy and reproducibility of CT volumetry with ImageJ. In accordance with the study by Wigmore et al., we found a ratio between liver weight and CT-measured liver volume of $0.88 \pm 0.04$ (SE) g/ml [6]. The most likely explanation of the systematic overestimation of liver volume compared to resected weight is that the three-dimensional reconstructions are based on a perfused liver, whereas the actual weight of the specimen was obtained directly after removal in a nonperfused state. Another explanation could be that the specific gravity of the liver is lower than $1 \mathrm{mg} / \mathrm{ml}$. However, the specific gravities of the various cellular and tissue components in the liver vary between $0.9 \mathrm{~g} / \mathrm{ml}$ (fat) and $1.10 \mathrm{mg} / \mathrm{ml}$ (hepatocytes) [11]. A specific gravity significantly $<1.0$ could only be explained by severe liver steatosis, which was found in only one of the resected specimens. Finally, aspiration of liver tissue by the Cavitron ultrasonic aspirator (CUSA) may contribute.

At present, the limits of liver resection are being increasingly explored. Indications for liver resection are continuously being extended; and former contraindications, such as bilobar disease, number of metastases, and even extrahepatic disease, have been abandoned in selected cases [12-14]. In general, radical tumor clearance with a 


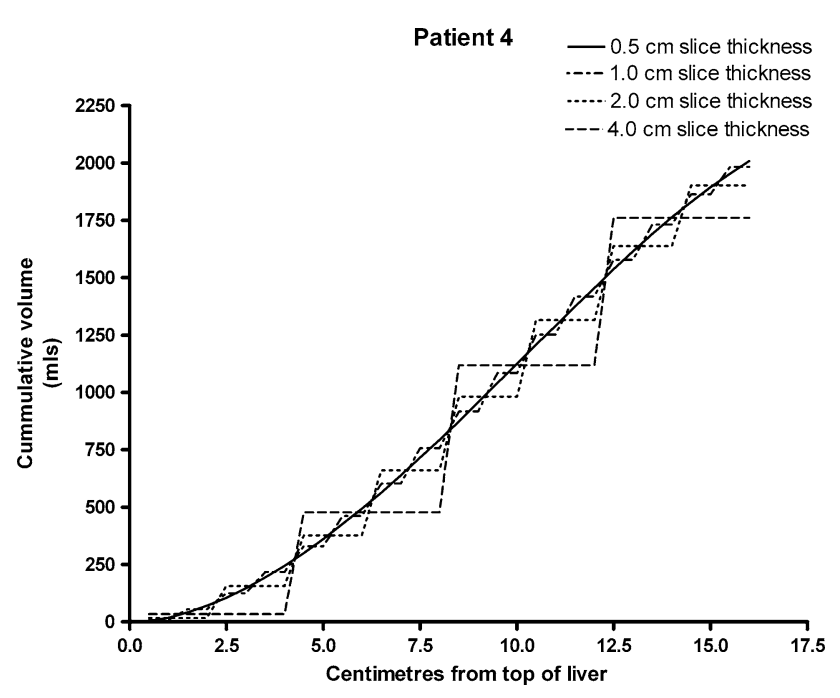

Fig. 4 Effect of increasing slice thickness on accuracy. It can be conceived clearly that a slice thickness of $\geq 2 \mathrm{~cm}$ leads to large deviations from the actual liver volume

minimal resection margin of at least $1 \mathrm{~mm}$ is sufficient to achieve a significant survival benefit [15]. Consequently, postoperative remnant liver volume and function have become the most important determinants of resectability. Repeat liver resections, more extensive resections, and resections after aggressive induction chemotherapy are likely to yield more patients with small remnant livers and more with postresectional liver failure.

The actual residual volume that limits resectability is still subject to debate, and the suggested minimal required remnant liver volume varies from $25 \%$ to $30 \%[3,4,16]$. In keeping with this, it has been shown that if meticulous attention is paid to the remnant liver volume and function, mortality can be decreased $[5,8,9]$. The variability in minimal required residual liver volume among individual patients is in all probability determined by the functional status of the parenchyma. It is common knowledge that major liver resection is less well tolerated in patients with cirrhosis [17] or steatosis of the liver [18]. Also, postchemotherapy nodular regenerative hyperplasia is considered not to have the same functional capacity as normal liver tissue. Consequently plain CT volumetry may not suffice to select patients for liver resection in these subgroups. True dynamic functional liver tests may become of additional use in these patients.

In this context, it is worthwhile to mention some recent reports using a combination of CT volumetric analysis and functional tests such as indocyanine green (ICG) clearance tests [19] or functional liver scintigraphy [20,21]. Equally, new methods become available to assess the role of the hepatic reticuloendothelial system and nitrogen clearance against the background of liver volumetry [22, 23]. The clinical applicability of such methods, however, awaits further confirmation.

A substantial number of patients admitted to specialized hepatobiliary units are referred from district general hospitals [24]. In our experience most of these patients already have a proper four-phase CT scan on CD-ROM from the referring institution. In most of these cases, however, it would not be possible to perform liver volumetry on these scans owing to incompatibility between radiologic software packages. ImageJ eliminates the necessity to perform an additional CT scan for the sole purpose of liver volumetry. Another advantage is that volumetry can be done by the surgeon without support from the radiology department. A potential disadvantage is the laboriousness of volumetry with ImageJ. Volumetric analyses takes on average $50 \pm 3$ seconds per slice. At a slice thickness of 5 $\mathrm{mm}$, a full volumetric analysis of a single liver requires on average 25 to 28 minutes for the entire liver. The time required to perform the measurement can be limited by increasing slice thickness and thereby reducing the number of CT slices that have to be outlined. However, an increase in slice thickness is accompanied by decreased accuracy of the measurements. The results show that a maximum CT slice thickness of $1.0 \mathrm{~cm}$ provides a good balance between accuracy and workload (Fig. 4). Time efficiency can be further improved by downloading a volume measurement "plug-in" that calculates the volume directly in the ROI manager. This eliminates the extra step of exporting calculated areas to Microsoft Excel. The plug-in can be downloaded freely from https://list.nih.gov/cgi-bin/ wa?A2=ind0202\&L=IMAGEJ\&P=R11200\&I=-3.

The accuracy of ImageJ to predict postoperative residual liver volume prospectively depends on the adherence to the intended resection line during the actual operation. In this retrospective study the transection plane could be carefully reconstructed based on the operation notes and guidance by the surgeons who performed the resection. The present study suggests that, in view of the excellent correlation between the predicted and actually resected weight, a reliable estimate of postoperative residual liver volume can be generated. All hepatectomies in the present study were anatomic resections. In the case of nonanatomical resection, it may be more difficult to predict the definitive transection plane and to outline it on a preoperative CT scan, but this problem would affect all software packages. Further studies are necessary to assess whether ImageJ provides the same volumetric accuracy for nonanatomic resections as for anatomic resections. In addition, evaluation of liver growth prior to repeated hepatectomy [25] or following other volume-enhancing procedures, such as portal vein embolization, would be interesting potential fields of application for ImageJ. 


\section{Conclusions}

ImageJ can be used for CT volumetric analysis of the liver on a personal computer. This application has the benefit that it is freely downloadable and brings CT volumetry to the surgeon's desktop independent of the support of a radiologist. Image J could be particularly useful in cases of tertiary referred patients who already have a proper CT scan on CD-ROM from the referring institution.

Acknowledgments This study was supported by grants from The Netherlands Organization for Health Research and Development to M.C.G.vdP. (920-03-317 AGIKO) and C.H.C.D. (907-00-033 Clinical Fellowship)

\section{References}

1. Jemal A, Murray T, Ward E, et al. (2005) Cancer statistics, 2005. CA Cancer J Clin; 55(1):10-30

2. Visser O, Siesling S, van Dijck J (2003) Incidence of cancer in The Netherlands 1999/2000. Vereniging van Integrale Kankercentra, Utrecht

3. Schindl MJ, Redhead DN, Fearon KC, et al. (2005) The value of residual liver volume as a predictor of hepatic dysfunction and infection after major liver resection. Gut 54(2):289-296

4. Shoup M, Gonen M, D’Angelica M, et al. (2003) Volumetric analysis predicts hepatic dysfunction in patients undergoing major liver resection. J Gastrointest Surg 7(3):325-330

5. Jarnagin WR, Gonen M, Fong Y, et al. (2002) Improvement in perioperative outcome after hepatic resection: analysis of 1,803 consecutive cases over the past decade. Ann Surg 236(4):397406; discussion 406-407

6. Wigmore SJ, Redhead DN, Yan XJ, et al. (2001) Virtual hepatic resection using three-dimensional reconstruction of helical computed tomography angioportograms. Ann Surg 233(2):221226

7. Lu Y, Wu Z, Liu C, Wang HH (2004) Hepatic volumetry with PhotoShop in personal computer. Hepatobiliary Pancreat Dis Int 3(1):82-85

8. Dimick JB, Cowan JA Jr, Knol JA, et al. (2003) Hepatic resection in the United States: indications, outcomes, and hospital procedural volumes from a nationally representative database. Arch Surg 138(2):185-191
9. Imamura H, Seyama Y, Kokudo N, et al. (2003) One thousand fifty-six hepatectomies without mortality in 8 years. Arch Surg 138(11):1198-1206; discussion 1206

10. Rasband WS (1997-2005) ImageJ. 1.33 edn. U.S. National Institutes of Health, Bethesda, MD

11. Alpini G, Phillips JO, Vroman B, et al. (1994) Recent advances in the isolation of liver cells. Hepatology 20(2):494-514

12. Shah SA, Haddad R, Al-Sukhni W, et al. (2006) Surgical resection of hepatic and pulmonary metastases from colorectal carcinoma. J Am Coll Surg 202(3):468-475

13. Fusai G, Davidson BR (2003) Management of colorectal liver metastases. Colorectal Dis 5(1):2-23

14. Scheele J, Altendorf-Hofmann A, Grube T, et al. (2001) [Resection of colorectal liver metastases: what prognostic factors determine patient selection?] Chirurg 72(5):547-560

15. Pawlik TM, Scoggins CR, Zorzi D, et al. (2005) Effect of surgical margin status on survival and site of recurrence after hepatic resection for colorectal metastases. Ann Surg 241(5):715-722, discussion $722-724$

16. Yigitler C, Farges O, Kianmanesh R, et al. (2003) The small remnant liver after major liver resection: how common and how relevant? Liver Transpl 9(9):S18-S25

17. Fan ST (2002) Methods and related drawbacks in the estimation of surgical risks in cirrhotic patients undergoing hepatectomy. Hepatogastroenterology 49(43):17-20

18. Behrns KE, Tsiotos GG, DeSouza NF, et al. (1998) Hepatic steatosis as a potential risk factor for major hepatic resection. $\mathrm{J}$ Gastrointest Surg 2(3):292-298

19. Fazakas J, Mandli T, Ther G, et al. (2006) Evaluation of liver function for hepatic resection. Transplant Proc 38(3):798-800

20. Satoh K, Yamamoto Y, Nishiyama Y, et al. (2003) ${ }^{99 \mathrm{~m}}$ Tc-GSA liver dynamic SPECT for the preoperative assessment of hepatectomy. Ann Nucl Med 17(1):61-67

21. Bennink RJ, Dinant S, Erdogan D, et al. (2004) Preoperative assessment of postoperative remnant liver function using hepatobiliary scintigraphy. J Nucl Med 45(6):965-971

22. Schindl MJ, Millar AM, Redhead DN, et al. (2006) The adaptive response of the reticuloendothelial system to major liver resection in humans. Ann Surg 243(4):507-514

23. Dejong CHC, Dowidar N, Wigmore SJ, et al. (2001)) Virtual hepatectomy and in vivo urea synthesis in patients with colorectal liver metastases. HPB 3(1):114 (abstract)

24. Majeed AW, Price C (2004) Resource and manpower calculations for the provision of hepatobiliary surgical services in the UK. Ann R Coll Surg Engl 86(2):91-95

25. Adam R, Laurent A, Azoulay D, et al. (2000) Two-stage hepatectomy: a planned strategy to treat irresectable liver tumors. Ann Surg 232(6):777-785 